\title{
Abstract Domains for Universal and Existential Properties
}

\author{
Andrew Heaton ${ }^{1}$, Patricia M. Hill ${ }^{2}$, and Andy King ${ }^{3}$ \\ 1 School of Computer Studies, University of Leeds, LS2 9JT, UK, \\ heaton@scs.leeds.ac.uk, \\ Tel: +44 113233 5322, Fax: +44 1132335468 . \\ 2 School of Computer Studies, University of Leeds, LS2 9JT, UK, \\ hill@scs.leeds.ac.uk. \\ 3 Computing Laboratory, University of Kent at Canterbury, CT2 7NF, UK, \\ amk@ukc.ac.uk.
}

\begin{abstract}
Abstract interpretation theory has successfully been used for constructing algorithms to statically determine run-time properties of programs. Central is the notion of an abstract domain, describing certain properties of interest about the program. In logic programming, program analyses typically fall into two different categories: either they detect program points where the property definitely holds (universal analyses) or possibly holds (existential analyses). We study the relation between such analyses in the case where the concrete domain is a lattice joingenerated by its set of join-irreducible elements. Although our intended application is for logic programming, the theory is sufficiently general for possible applications to other languages.
\end{abstract}

\section{Introduction}

Abstract interpretation theory has successfully been used for constructing algorithms to statically determine run-time properties of programs. Traditionally, the semantics of the program is specified with a concrete domain. The central notion is to approximate program semantics by defining an abstract domain whose operations mimic those of the concrete domain. The abstract domain describes certain properties of interest about the program. Each element of the abstract domain specifies information about a possibly infinite number of concrete states. Thus, in order to construct an abstract domain tracing a property of the program, the property needs to be considered as a property over sets of concrete states.

Our aim is to provide new techniques for the construction of new abstract domains from given ones. Many operations have been designed for systematically constructing new domains. Domain operators studied include reduced product [8,4], reduced power [8] and disjunctive completion [8,11]. Linear refinement is introduced in [13] as an extension of the Heyting completion studied in [14]. In [15], a new domain for freeness analysis of logic programs is defined using linear 
refinement. In this paper, we suppose that the concrete domain is a lattice joingenerated by its set of join-irreducible elements. In this case, given any property $p$ defined over each individual concrete state, $p$ can always be uniformly extended to a property over sets of concrete states.

For example, in logic programming it is standard to define the concrete domain as the powerset of substitutions, $\wp(S u b)$, partially ordered by set inclusion. $\wp(S u b)$ is join-generated by $S u b$. For many properties of logic programs, it is natural to first define the property on substitutions and then lift the property to include sets of substitutions. Consider the property of groundness. A variable $x$ is ground under a substitution $\theta \in S u b$ if $\theta$ binds $x$ to a term with no variables. Letting $X$ be the set of variables of interest, the mapping $g r: S u b \rightarrow \wp(X)$ is defined:

$$
g r(\theta)=\{x \in X \mid \operatorname{var}(\theta(x))=\emptyset\}
$$

Suppose we now want to consider groundness as a property with domain $\wp(S u b)$. We can consider either definite (universal) groundness or possible (existential) groundness. For definite groundness, $G r^{\forall}: \wp(S u b) \rightarrow \wp(X)$ is defined:

$$
G r^{\forall}(\Theta)=\bigcap\{g r(\theta) \mid \theta \in \Theta\} .
$$

For possible groundness, $G r^{\exists}: \wp(S u b) \rightarrow \wp(X)$ is defined:

$$
G r^{\exists}(\Theta)=\bigcup\{g r(\theta) \mid \theta \in \Theta\} .
$$

Note that definite groundness traces positive information about the groundness of program variables, whereas possible groundness traces negative information. Knowledge of both positive and negative information about program properties such as groundness is particularly useful for debugging applications.

In general, given a concrete domain $C$, an abstract domain $D$ and a property $p$ mapping the join-irreducible elements of $C$ to $D, p$ is extended to $C$ using the join operation of $D$. We name this extension of $p$ the $D$-lattice property of $p$. For example, $G r^{\forall}$ is the $D_{g r}^{\forall}$-lattice property of $g r$ where $D_{g r}^{\forall}$ is the lattice $\wp(S u b)$, partially ordered by $\supseteq$ with set intersection as the join operation. $G r^{\exists}$ is the $D_{g r}^{\exists}$-lattice property of $g r$ where $D_{g r}^{\exists}$ is the lattice $\wp(S u b)$, partially ordered by $\subseteq$ with set union as the join operation.

The main theoretical results shown are as follows:

- Given a Galois connection $(C, \alpha, D, \gamma)$ (where $C$ is completely distributive and join-generated by its set of join-irreducible elements) specifying an analysis tracing positive information of $p$, we show how to construct a mirror Galois connection $\left(C, \alpha^{m}, D^{d}, \gamma^{m}\right.$ ) (where $D^{d}$ is the dual lattice of $D$ ) specifying an analysis tracing negative information of $p$.

- Suppose op :C $\rightarrow C$ is a concrete operation and $\left\langle D, o p^{\prime}\right\rangle$ is a correct abstract interpretation of $\langle C, o p\rangle$ specified by $(C, \alpha, D, \gamma)$. We find conditions on $\left\langle D, o p^{\prime}\right\rangle$ and $\langle C, o p\rangle$ which ensure that $\left\langle D^{d}, o p^{\prime}\right\rangle$ is a correct abstract interpretation of $\langle C, o p\rangle$ specified by $\left(C, \alpha^{m}, D^{d}, \gamma^{m}\right)$. 
The paper is organised as follows: in Section 3 we define the notion of lattice properties and mirror properties. Section 4 considers some applications with the well-known domains Pos and Sharing of logic programming. In Section 5 we consider the safe approximation of concrete functions in analyses for mirror properties. Finally, Section 6 gives some concluding remarks and directions for future work.

\section{Preliminaries}

Throughout the paper, we assume familiarity with the basic notions of lattice

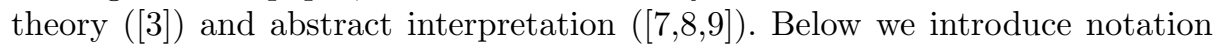
and recall some of the central notions.

\subsection{Lattice Theory}

In the following, we assume $\left\langle A, \sqsubseteq_{A}, \sqcap_{A}, \sqcup_{A}, \top_{A}, \perp_{A}\right\rangle$ is a complete lattice. The dual lattice $\left\langle A, \sqsubseteq_{A}^{d}, \sqcap_{A}^{d}, \sqcup_{A}^{d}, \top_{A}^{d}, \perp_{A}^{d}\right\rangle$ is defined such that:

1. $\forall a, b \in A . a \sqsubseteq_{A}^{d} b$ iff $b \sqsubseteq_{A} a$;

2. $\sqcap_{A}^{d}=\sqcup_{A}$;

3. $\sqcup_{A}^{d}=\Pi_{A}$;

4. $\top_{A}^{d}=\perp_{A}$;

5. $\perp_{A}^{A}=\top_{A}$.

We will often write $A^{d}$ to denote the dual lattice $\left\langle A, \sqsubseteq_{A}^{d}, \sqcap_{A}^{d}, \sqcup_{A}^{d}, \top_{A}^{d}, \perp_{A}^{d}\right\rangle$. Given a mapping $f: A_{1} \rightarrow A_{2}$, we will sometimes abuse notation by also writing $f$ to denote the dual mapping $f^{d}: A_{1}^{d} \rightarrow A_{2}^{d}$ such that $f(a)=f^{d}(a)$ for all $a \in A_{1}$.

An element $a \in A$ is join-irreducible if, for any $S \subseteq A, a=\sqcup_{A} S$ implies $a \in S$. The set of join-irreducible elements of $A$ is denoted by $J I(A)$. Letting $S \subseteq A$, then $A$ is join-generated by $S$ if, for all $a \in A, a=\sqcup_{A}\left\{x \in S \mid x \sqsubset_{A} a\right\}$. For convenience, we assume $\perp_{A}=\sqcup_{A} \emptyset$. An element $a \in A$ is an atom if a covers $\perp_{A}$, i.e. $a \neq \perp_{A}$ and $\forall x \in A$. $\left(\perp_{A} \sqsubset_{A} x \sqsubseteq_{A} a\right) \Rightarrow(x=a)$. We denote by atom $_{A}$ the set of atoms of $A$. Note that atom $A \subseteq J I(A)$. A is atomistic if $A$ is join-generated by atom $_{A}$. $A$ is dual-atomistic if $\bar{A}^{d}$ is atomistic.

A complete lattice $A$ is completely distributive if, for any $\left\{x_{i, k} \mid i \in I, k \in\right.$ $K(i)\} \subseteq A$, the following identity holds:

$$
\prod_{i \in I} \bigsqcup_{k \in K(i)} x_{i, k}=\bigsqcup_{f \in I \rightsquigarrow K} \prod_{i \in I} x_{i, f(i)},
$$

where for any $i \in I, K(i)$ is a set of indices, and $I \rightsquigarrow K$ is the set of all functions $f$ from $I$ to $\bigcup_{i \in I} K(i)$ such that $\forall i \in I . f(i) \in K(i)$.

Example 1. The powerset of any set $S, \wp(S)$, ordered with set-theoretic inclusion, is completely distributive and join-generated by $S$. In this case $\wp(S)$ is also an atomistic lattice where the atoms are the elements of $S$. 
The key property of completely distributive lattices we shall use is:

Lemma 1 ([2]). Let $A$ be a completely distributive lattice. Then, $x \in J I(A)$ iff for any $S \subseteq A, x \sqsubseteq_{A} \bigsqcup_{A} S$ implies $x \sqsubseteq_{A} s$ for some $s \in S$.

\subsection{Galois Connections}

If $C$ and $D$ are posets and $\alpha: C \rightarrow D, \gamma: D \rightarrow C$ functions such that $\forall c \in C . \forall d \in D . \alpha(c) \sqsubseteq D d \Leftrightarrow c \sqsubseteq C \gamma(d)$, then $(C, \alpha, D, \gamma)$ is a Galois connection between $C$ and $D$. If in addition $\gamma$ is 1-1, or, equivalently, $\alpha$ is onto then $(C, \alpha, D, \gamma)$ is a Galois insertion of $D$ in $C$. In the setting of abstract interpretation, $C$ and $D$ are called the concrete and abstract domains, respectively. Given a Galois connection $(C, \alpha, D, \gamma), \alpha$ and $\gamma$ are uniquely determined by each other. A practical consequence of this is that an abstract interpretation can be performed by defining only one of $\alpha$ or $\gamma$. We assume that every concrete domain $C$ and abstract domain $D$ form complete lattices. Given a concrete domain $C$ and an abstract domain $D$, a property is defined as a (partial) mapping from $C$ to $D$. Every Galois connection $(C, \alpha, D, \gamma)$ can be viewed as a specification of the property $\alpha: C \rightarrow D$.

An important property of Galois connections is the preservation of bounds. Suppose $C, D$ are complete lattices. A mapping $\alpha: C \rightarrow D$ is additive if it preserves least upper bounds. Thus if $S \subseteq C$ then $\alpha\left(\bigsqcup_{C} S\right)=\bigsqcup_{D}\{\alpha(c) \mid c \in S\}$. A mapping $\alpha: C \rightarrow D$ is co-additive if $\alpha: C^{d} \rightarrow D^{d}$ is additive. If $(C, \alpha, D, \gamma)$ is a Galois connection, then $\alpha$ is additive. The converse is also true, i.e. if $\alpha$ is additive then $\alpha$ entirely determines a unique Galois connection $(C, \alpha, D, \gamma)$. Thus in order to define a Galois connection $(C, \alpha, D, \gamma)$ (where $C, D$ are complete lattices), it is sufficient to define an additive $\alpha$.

One way of defining new Galois connections is by composition. Given two Galois connections $\left(C, \alpha_{A}, A, \gamma_{A}\right)$ and $\left(A, \alpha_{D}, D, \gamma_{D}\right),\left(C, \alpha_{A} \circ \alpha_{D}, D, \gamma_{D} \circ \gamma_{A}\right)$ is a Galois connection. We call $\left(C, \alpha_{A} \circ \alpha_{D}, D, \gamma_{D} \circ \gamma_{A}\right)$ the composition of $\left(C, \alpha_{A}, A, \gamma_{A}\right)$ and $\left(A, \alpha_{D}, D, \gamma_{D}\right)$.

Suppose $(C, \alpha, D, \gamma)$ is a Galois connection and op $_{C}: C \rightarrow C$, op $_{D}: D \rightarrow D$ are operations on $C$ and $D$, respectively. $\left\langle D, o p_{D}\right\rangle$ is a correct abstract interpretation of $\left\langle C, o p_{C}\right\rangle$ specified by $(C, \alpha, D, \gamma)$ if $\alpha\left(o p_{C}(\gamma(d))\right) \sqsubseteq_{D} o p_{D}(d)$ for all $d \in D .\left\langle D, o p_{D}\right\rangle$ is optimal if $o p_{D}=\alpha \circ o p_{C} \circ \gamma$. If $\left\langle D, o p_{D}\right\rangle$ is optimal, then $o p_{D}$ is the best approximation of $o p_{C}$ relative to $D .\left\langle D, o p_{D}\right\rangle$ is complete if $\alpha \circ o p_{C}=o p_{D} \circ \alpha$. Completeness is a stronger property than optimality. Indeed, whenever $\left\langle D, o p_{D}\right\rangle$ is complete, it can be shown that $o p_{D}=\alpha \circ o p_{C} \circ \gamma[1012$. The completeness of $o p_{C}$ depends on $D$ and is a property of the abstract domain.

If $(C, \alpha, D, \gamma)$ is a Galois insertion, each value of the abstract domain $D$ is useful in the presentation of the concrete domain as all the elements of $D$ represent distinct members of $C$. Moreover, any Galois connection may be lifted to a Galois insertion. This is done by identifying those values of the abstract domain with the same concrete meaning into an equivalence class. This process is known as reduction of the abstract domain. Each Galois insertion $(C, \alpha, D, \gamma)$ can equivalently be considered as an upper closure operator on $C, \rho=\gamma \circ \alpha$. For 
every Galois connection $(C, \alpha, D, \gamma)$, let $\left(C, \alpha_{\equiv}, D_{\equiv}, \gamma_{\equiv}\right)$ be the Galois insertion obtained by reducing $(C, \alpha, D, \gamma)$. We associate the (upper) closure operator $\rho=\gamma_{\equiv} \circ \alpha \equiv$ with $(C, \alpha, D, \gamma)$. The set of closure operators on $C$ is partially ordered such that $\rho_{1} \sqsubseteq \rho_{2}$ if $\forall c \in C . \rho_{1}(c) \sqsubseteq C \rho_{2}(c)$. In this approach, the order relation on the set of closure operators on $C$ corresponds to the order by means of which abstract domains are compared with regard to precision. More formally, if $\left(C, \alpha_{1}, D_{1}, \gamma_{1}\right)$ and $\left(C, \alpha_{2}, D_{2}, \gamma_{2}\right)$ are Galois connections with the associated closure operators $\rho_{1}$ and $\rho_{2}$, respectively, then we say $D_{1}$ is more precise than $D_{2}$ if $\rho_{1} \sqsubseteq \rho_{2}$.

\section{Properties of Programs}

In abstract interpretation, Galois connections are used to specify properties of programs. To define a Galois connection $(C, \alpha, D, \gamma)$ between a concrete domain $C$ and an abstract domain $D$, all we need to do is define an additive function $\alpha: C \rightarrow D$. It is well known that in the case where the concrete lattice $C$ is join-generated by $J I(C)$, additive functions mapping $C$ to an abstract domain $D$ are completely determined by their values for join irreducible elements. More specifically, if $\alpha: C \rightarrow D$ is additive then

$$
\alpha(c)=\bigsqcup_{D}\{\alpha(x) \mid x \in J I(C) \wedge x \sqsubseteq C c\} .
$$

Example 2. For logic programs, a standard choice of concrete lattice is the atomistic lattice $C_{L}=\langle\wp(S u b), \subseteq, \cap, \cup, \emptyset, S u b\rangle$, where $S u b$ denotes the set of idempotent substitutions.

A program variable is ground if it is bound to a unique value. Groundness can be thought of as a property over $S u b$, i.e. as a property over $J I\left(C_{L}\right)$. Let $X$ be the set of variables of interest. Then the set of variables ground under $\theta \in S u b$ is given by $g r: J I\left(C_{L}\right) \rightarrow \wp(X)$ defined

$$
\operatorname{gr}(\theta)=\{x \in X \mid \operatorname{var}(\theta(x))=\emptyset\} .
$$

Let $\Theta \subseteq S u b$. The set of variables that are definitely ground under all $\theta \in \Theta$ is given by $G r^{\forall}: C_{L} \rightarrow \wp(X)$ where

$$
G r^{\forall}(\Theta)=\{x \in X \mid \forall \theta \in \Theta \cdot \operatorname{var}(\theta(x))=\emptyset\}=\bigcap\{g r(\theta) \mid \theta \in \Theta\} .
$$

Alternatively, the set of variables that are possibly ground under all $\theta \in \Theta$ is given by $G r^{\exists}: C_{L} \rightarrow \wp(X)$ where

$$
G r^{\exists}(\Theta)=\{x \in X \mid \exists \theta \in \Theta \cdot \operatorname{var}(\theta(x))=\emptyset\}=\bigcup\{g r(\theta) \mid \theta \in \Theta\} .
$$

Definition 1. Let $C$ be a lattice. Then $p$ is an $J I$ property for $C$ if there exists a set $D$ such that $p$ maps $J I(C)$ to $D($ denoted $p: J I(C) \rightarrow D)$. 
Definition 2. Suppose $C$ is join-generated by $J I(C)$ and let $p: J I(C) \rightarrow D$ be a $J I$ property for $C$. Suppose $D$ forms a complete lattice under the partial ordering $\sqsubseteq_{D}$. Then the $D$-lattice property of $p, P: C \rightarrow D$, is defined such that for every $c \in C$,

$$
P(c)=\bigsqcup_{D}\left\{p(x) \mid x \in J I(C) \wedge x \sqsubseteq_{C} c\right\} .
$$

Let $D^{d}$ be the dual lattice of $D$. If $P$ is the $D$-lattice property of $p$ then we define the mirror property of $P$ to be the $D^{d}$-lattice property of $p$.

Note that the mirror of the mirror of $P$ is $P$.

Example 3. Let $D_{g r}$ be the complete lattice $(\wp(X), \subseteq, \cap, \cup, \emptyset, X)$. In Example 2 $G r^{\exists}$ is the $D_{g r}$-lattice property of $g r$, and $G r^{\forall}$ is the $D_{g r}^{d}$-lattice property of $g r$. Hence $G r^{\forall}$ and $G r^{\exists}$ are mirror properties.

In the case where $C$ is also a completely distributive lattice, we have the following theorem.

Theorem 1. Suppose $C$ is a completely distributive lattice join generated by $J I(C)$ and $D$ is a complete lattice. Let $(C, \alpha, D, \gamma)$ be a Galois connection. Then there exists $\alpha^{m}, \gamma^{m}$ such that

1. $\alpha^{m}$ is the mirror property of $\alpha$.

2. $\left(C, \alpha^{m}, D^{d}, \gamma^{m}\right)$ is a Galois connection.

Proof. To prove 1, observe that as $C$ is join-generated by $J I(C)$, for each $c \in C$,

$$
\alpha(c)=\bigsqcup_{D}\left\{\alpha(x) \mid x \in J I(C) \wedge x \sqsubseteq_{C} c\right\} .
$$

Hence by Definition 2

$$
\alpha^{m}(c)=\prod_{D}\left\{\alpha(x) \mid x \in J I(C) \wedge x \sqsubseteq_{C} c\right\} .
$$

To prove 2 , it is sufficient to show that $\alpha^{m}$ is additive. But

$$
\begin{array}{rlrl}
\alpha^{m}\left(\bigsqcup_{C} S\right) & =\prod_{D}\left\{\alpha(x) \mid x \in J I(C) \wedge x \sqsubseteq_{C} \bigsqcup_{C} S\right\} \quad & \text { (by Definition 2) } \\
& =\prod_{D}\left\{\alpha(x) \mid x \in J I(C) \wedge x \sqsubseteq_{C} s \wedge s \in S\right\} \quad \text { (by Lemma 1) } \\
& =\prod_{D}\left\{\alpha^{m}(s) \mid s \in S\right\} .
\end{array}
$$

Hence $\alpha^{m}$ is additive.

The compositional design of Galois connections is a method for specifying program properties by successive refinements. The following lemma gives a sufficient condition for the preservation of compositions of Galois connections between mirror properties. 
Lemma 2. Suppose $C$ is a completely distributive lattice join-generated by $J I(C)$, and $A, D$ are complete lattices. Suppose $\left(C, \alpha_{p}, D, \gamma_{p}\right),\left(C, \alpha_{p}^{m}, D^{d}, \gamma_{p}^{m}\right)$, $\left(C, \alpha_{A}, A, \gamma_{A}\right)$ and $\left(C, \alpha_{A}^{m}, A^{d}, \gamma_{A}^{m}\right)$ are Galois connections such that $\alpha_{p}, \alpha_{p}^{m}$ and $\alpha_{A}, \alpha_{A}^{m}$ are mirror properties. Also suppose $\left(A, \alpha_{D}, D, \gamma_{D}\right)$ is a Galois connection such that $\left(C, \alpha_{p}, D, \gamma_{p}\right)$ is the composition of $\left(C, \alpha_{A}, A, \gamma_{A}\right)$ and $\left(A, \alpha_{D}, D, \gamma_{D}\right)$. Then if $\alpha_{D}$ is co-additive, there exists $\gamma_{D}: D^{d} \rightarrow A^{d}$ such that $\left(A^{d}, \alpha_{D}, D^{d}, \gamma_{D}\right)$ forms a Galois connection and $\left(C, \alpha_{p}^{m}, D^{d}, \gamma_{p}^{m}\right)$ is the composition of $\left(C, \alpha_{A}^{m}, A^{d}, \gamma_{A}^{m}\right)$ and $\left(A^{d}, \alpha_{D}, D^{d}, \gamma_{D}\right)$.

Proof. First note that $\alpha_{D}: A \rightarrow D$ is co-additive implies that $\alpha_{D}: A^{d} \rightarrow D^{d}$ is additive, and so there exists $\gamma_{D}: D^{d} \rightarrow A^{d}$ such that $\left(A^{d}, \alpha_{D}, D^{d}, \gamma_{D}\right)$ forms a Galois connection.

To show that $\left(C, \alpha_{p}^{m}, D^{d}, \gamma_{p}^{m}\right)$ is the composition of $\left(C, \alpha_{A}^{m}, A^{d}, \gamma_{A}^{m}\right)$ and $\left(A^{d}, \alpha_{D}, D^{d}, \gamma_{D}\right)$, it is sufficient to show that $\alpha_{p}^{m}=\alpha_{D} \circ \alpha_{A}^{m}$. Suppose $c \in C$. By Definition 2,

$$
\alpha_{p}^{m}(c)=\prod_{D}\left\{\alpha_{p}(x) \mid x \in J I(C) \wedge x \sqsubseteq_{C} c\right\} .
$$

Now $\alpha_{p}(x)=\alpha_{D}\left(\alpha_{A}(x)\right)$ and so

$$
\alpha_{p}^{m}(c)=\prod_{D}\left\{\alpha_{D}\left(\alpha_{A}(x)\right) \mid x \in J I(C) \wedge x \sqsubseteq_{C} c\right\} .
$$

But $\alpha_{D}$ is co-additive and so

$$
\alpha_{p}^{m}(c)=\alpha_{D}\left(\prod_{A}\left\{\alpha_{A}(x) \mid x \in J I(C) \wedge x \sqsubseteq_{C} c\right\}\right)=\alpha_{D}\left(\alpha_{A}^{m}(c)\right) . \square
$$

Let $\rho_{p}, \rho_{A}$ be the associated closure operators of $\left(C, \alpha_{p}, D, \gamma_{p}\right)$ and $\left(C, \alpha_{A}, A, \gamma_{A}\right)$, respectively. Note that whenever $\left(C, \alpha_{p}, D, \gamma_{p}\right)$ is the composition of $\left(C, \alpha_{A}, A, \gamma_{A}\right)$ and $\left(A, \alpha_{D}, D, \gamma_{D}\right)$, then $\rho_{A} \sqsubseteq \rho_{p}$. Thus Lemma 2 can be interpreted as giving a sufficient condition for the preservation of the relative precision between mirror properties, that is, when $\rho_{A} \sqsubseteq \rho_{p}$ implies $\rho_{A}^{m} \sqsubseteq \rho_{p}^{m}$ (where $\rho_{p}^{m}, \rho_{A}^{m}$ are the associated closure operators of $\left(C, \alpha_{A}^{m}, A^{d}, \gamma_{A}^{m}\right)$ and $\left(C, \alpha_{p}^{m}, D^{d}, \gamma_{p}^{m}\right)$, respectively).

\section{Applications}

We consider the abstract domains Pos and Sharing from logic programming. In the following, let Vars denote a countable set of variables, and $X$ denote a non-empty finite subset of Vars containing the variables of interest.

\subsection{Pos}

We briefly recall the definition of Pos. The domain Pos consists of the set of positive propositional formulae on $X$, where a propositional formula is positive 
if it is satisfied when every variable is assigned the value true. Pos is a lattice whose ordering is given by logical consequence, and the join and meet by logical disjunction and conjunction, respectively. Adding the bottom propositional formula false to Pos, makes Pos a complete lattice. Letting $C_{L}$ be the concrete domain defined in Example 2, the Galois insertion $\left(C_{L}, \alpha_{p o s}, P o s, \gamma_{p o s}\right)$ is such that $\alpha_{\text {pos }}: C_{L} \rightarrow$ Pos where for all $\Theta \in C_{L}$,

$$
\alpha_{\text {pos }}(\Theta)=\bigvee_{\theta \in \Theta} \bigwedge_{x \in X}\{x \leftrightarrow \bigwedge \operatorname{var}(\theta(x))\}
$$

Note that $\alpha_{\text {pos }}$ is the Pos-lattice property of the $J I$ property $p_{\text {pos }}: S u b \rightarrow$ Pos defined such that

$$
p_{\text {pos }}(\theta)=\bigwedge_{x \in X}\{x \leftrightarrow \bigwedge \operatorname{var}(\theta(x))\} .
$$

The abstract unification function for Pos, Unif ${ }^{\text {pos }}:$ Pos $\times$ Pos $\rightarrow$ Pos, is given by logical conjunction, that is, the meet operation of Pos.

Recall that in Examples 2 and 3 ] definite groundness is specified by $G r^{\forall}$. In fact $G r^{\forall}$ maps $C_{L}$ onto $D_{g r}^{d}$ and so there exists $\gamma^{\forall}$ such that $\left(C_{L}, G r^{\forall}, D_{g r}^{d}, \gamma^{\forall}\right)$ forms a Galois insertion. This domain is originally due to Jones and Søndergaard [16]. In [18, when considering the concrete domain to be sets of substitutions closed by instantiation, it is shown that Pos can be constructed by using only the definition of groundness. More specifically, [18] shows that Pos is exactly the least abstract domain which contains all the (double) intuitionistic implications between elements of $D_{g r}^{d}$.

Let $\alpha_{D}:$ Pos $\rightarrow D_{g r}^{d}$ be defined such that for all $\phi \in P o s$,

$$
\alpha_{D}(\phi)=\{x \in X \mid \phi \models x\} .
$$

Now $\alpha_{D}$ is additive since $\alpha_{D}\left(\phi_{1} \vee \phi_{2}\right)=\alpha_{D}\left(\phi_{1}\right) \cap \alpha_{D}\left(\phi_{2}\right)$. Hence there exists $\gamma_{D}$ such that $\left(P o s, \alpha_{D}, D_{g r}^{d}, \gamma_{D}\right)$ forms a Galois connection. Also $G r^{\forall}(\Theta)=$ $\alpha_{D}\left(\alpha_{\text {pos }}(\Theta)\right)$ for all $\Theta \in C_{L}$, therefore $\left(C_{L}, G r^{\forall}, D_{g r}^{d}, \gamma^{\forall}\right)$ is the composition of $\left(C_{L}, \alpha_{\text {pos }}\right.$, Pos,$\left.\gamma_{\text {pos }}\right)$ and $\left(\right.$ Pos $\left., \alpha_{D}, D_{g r}^{d}, \gamma_{D}\right)$.

The mirror property of $G r^{\forall}$ is $G r^{\exists}$. Now $G r^{\exists}$ maps $C_{L}$ onto $D_{g r}$ and so there exists $\gamma^{\exists}$ such that $\left(C_{L}, G r^{\exists}, D_{g r}^{d}, \gamma^{\exists}\right)$ forms a Galois insertion.

The mirror property of $\alpha_{\text {pos }}$ is $\alpha_{\text {pos }}^{m}: C_{L} \rightarrow \operatorname{Pos}^{d}$ where

$$
\alpha_{p o s}^{m}(\Theta)=\bigwedge_{\theta \in \Theta} \bigwedge_{x \in X}\{x \leftrightarrow \bigwedge \operatorname{var}(\theta(x))\}
$$

Lemma 3. There exists $\gamma_{\text {pos }}^{m}$ such that $\left(C_{L}, \alpha_{\text {pos }}^{m}, P o s^{d}, \gamma_{\text {pos }}^{m}\right)$ forms a Galois connection. Also $\left(C_{L}, G r^{\exists}, D_{g r}, \gamma^{\exists}\right)$ is the composition of $\left(C_{L}, \alpha_{\text {pos }}^{m}, P_{o s}^{d}, \gamma_{\text {pos }}^{m}\right)$ and $\left(P_{o s}{ }^{d}, \alpha_{D}, D_{g r}, \gamma_{D}\right)$.

Proof. By Theorem 1 there exists $\gamma_{\text {pos }}^{m}$ such that $\left(C_{L}, \alpha_{\text {pos }}^{m}, P o s^{d}, \gamma_{\text {pos }}^{m}\right)$ forms a Galois connection. Now $\alpha_{D}(\phi \wedge \psi)=\alpha_{D}(\phi) \cup \alpha_{D}(\psi)$, and so $\alpha_{D}:$ Pos $\rightarrow D_{g r}^{d}$ is co-additive. Therefore by Lemma 2 ( $\left.C_{L}, G r^{\exists}, D_{g r}, \gamma^{\exists}\right)$ is the composition of $\left(C_{L}, \alpha_{\text {pos }}^{m}\right.$, Pos $\left.^{d}, \gamma_{\text {pos }}^{m}\right)$ and $\left(\right.$ Pos $\left.^{d}, \alpha_{D}, D_{g r}, \gamma_{D}\right)$. 
Lemma 4. If $\operatorname{Card}(X) \geq 2, \alpha_{\text {pos }}^{m}$ is not onto, thus $\left(C_{L}, \alpha_{\text {pos }}^{m}, \operatorname{Pos}^{d}, \gamma_{\text {pos }}^{m}\right)$ is not a Galois insertion.

Proof. By inspecting the definition of $\alpha_{\text {pos }}^{m}$, it can be seen that $\alpha_{\text {pos }}^{m}(\Theta) \neq \bigvee X$ when $\operatorname{Card}(X) \geq 2$, for any $\Theta \in C_{L}$. Hence $\alpha_{\text {pos }}^{m}$ is not onto.

In order to obtain a Galois insertion, we apply the reduction process to $\operatorname{Pos}^{d}$. $\left(C_{L}, \alpha_{\text {pos }}^{m}, \operatorname{Pos}^{d}, \gamma_{\text {pos }}^{m}\right)$ reduces to $\left(C_{L}, \alpha_{\text {pos } / \equiv}^{m}, \operatorname{Pos}^{d} / \equiv, \gamma_{\text {pos } / \equiv}^{m}\right)$ where for $\phi, \psi \in$ Pos ${ }^{d}$

$$
\phi \equiv \psi \Leftrightarrow \gamma_{\text {pos }}^{m}(\phi)=\gamma_{\text {pos }}^{m}(\psi), \quad \alpha_{\text {pos } / \equiv}^{m}(c)=\left\{\phi \mid \phi \equiv \alpha_{\text {pos }}^{m}(c)\right\}
$$

Let $\Gamma \subseteq \operatorname{Pos}^{d}$ be defined such that

$$
\Gamma=\left\{x \leftrightarrow \bigwedge\left\{y_{1}, \ldots, y_{n}\right\} \mid \forall 1 \leq i \leq n . x \neq y_{i}\right\}
$$

By inspecting the definition of $\alpha_{\text {pos }}^{m}$ (and noting that $S u b$ is the set of idempotent substitutions, i.e. $\theta \in S u b$ implies $x \notin \operatorname{var}(\theta(x))$ for all $x)$, it can be seen that $\operatorname{Pos}^{d} / \equiv$ is the lattice $\Lambda \subseteq \operatorname{Pos}^{d}$ where $\Lambda$ is the closure of $\Gamma$ under conjunction. From Lemma 3 we obtain:

Theorem 2. $\operatorname{Pos}^{d} / \equiv$ is more precise than $D_{g r}$.

Thus the precision ordering has been preserved for the mirror properties.

\subsection{Sharing}

We define Sharing as in [1]. We define the set sharing domain $S H=\wp(S G)$ where $S G=\{S \subseteq \wp(X) \mid \emptyset \notin S\}$. SH is partially ordered by set inclusion such that the join is given by set union and the meet by set intersection.

Let $C_{L}$ be the concrete domain defined in Example 2. The set of variables occurring in a substitution $\theta$ through the variable $v$ is given by the mapping occs $: S u b \times X \rightarrow \wp(X)$ defined such that

$$
\operatorname{occs}(\theta, x)=\{y \in X \mid x \in \operatorname{var}(\theta(y))\} .
$$

Given this, the Galois insertion $\left(C_{L}, \alpha_{s h}, S H, \gamma_{s h}\right)$ specifying $S H$ can be defined such that

$$
\alpha_{s h}(\Theta)=\bigcup_{\theta \in \Theta}\{\operatorname{occs}(\theta, x) \mid x \in \operatorname{Vars}, \operatorname{occs}(\theta, x) \neq \emptyset\} .
$$

Note that $\alpha_{s h}$ is the $S H$-lattice property of the $J I$ property $p_{s h}: S u b \rightarrow S H$ defined such that

$$
p_{s h}(\theta)=\{\operatorname{occs}(\theta, x) \mid x \in \operatorname{Vars}, \operatorname{occs}(\theta, x) \neq \emptyset\} .
$$

For Sharing, the abstract unification function is defined as a mapping which captures the effects of a binding $x \rightarrow t$ on an element of $S H$. The definition uses the following three operations defined over $S H$. 
The function bin : $S H \times S H \rightarrow S H$, called binary union is given by

$$
\operatorname{bin}\left(S_{1}, S_{2}\right)=\left\{s_{1} \cup s_{2} \mid s_{1} \in S_{1}, s_{2} \in S_{2}\right\} .
$$

The star-union function $(\cdot)^{*}: S H \rightarrow S H$ is given by

$$
S^{*}=\left\{s \in S G \mid \exists S^{\prime} \subseteq S . s=\bigcup S^{\prime}\right\} .
$$

The relevant component function $\mathrm{rel}: \wp(X) \times S H \rightarrow S H$ is given by

$$
\operatorname{rel}(V, S)=\{s \in S \mid s \cap V \neq \emptyset\} .
$$

Let $v_{x}=\{x\}, v_{t}=\operatorname{var}(t)$ and $v_{x t}=v_{x} \cup v_{t}$. Then

$$
U n i f^{s h}(S, x \rightarrow t)=\left(S \backslash\left(\operatorname{rel}\left(v_{x t}, S\right)\right) \cup \operatorname{bin}\left(\operatorname{rel}\left(v_{x}, S\right)^{*}, \operatorname{rel}\left(v_{t}, S\right)^{*}\right) .\right.
$$

A domain for pair sharing is $P S=\wp(\operatorname{Pairs}(X))$ where $\operatorname{Pairs}(X)=\{\{x, y\} \mid$ $x, y \in X, x \neq y\}$. PS is specified by the Galois insertion $\left(C_{L}, \alpha_{p s}, P S, \gamma_{p s}\right)$, where

$$
\alpha_{p s}(\Theta)=\bigcup_{\theta \in \Theta}\{\{x, y\} \in \operatorname{Pairs}(X) \mid \operatorname{var}(\theta(x)) \cap \operatorname{var}(\theta(y)) \neq \emptyset\} .
$$

Note that $\alpha_{p s}$ is the $P S$-lattice property of the $J I$ property $p_{p s}: S u b \rightarrow P S$ defined such that

$$
p_{p s}(\theta)=\{\{x, y\} \in \operatorname{Pairs}(X) \mid \operatorname{var}(\theta(x)) \cap \operatorname{var}(\theta(y)) \neq \emptyset\} .
$$

Defining $\alpha_{s p}: S H \rightarrow P S$ such that

$$
\alpha_{s p}(S)=\bigcup\{\operatorname{Pairs}(s) \mid s \in S\},
$$

it follows that $\alpha_{p s}(\Theta)=\alpha_{s p}\left(\alpha_{s h}(\Theta)\right)$ for all $\Theta \in C_{L}$. Also $\alpha_{s p}\left(S_{1} \cup S_{2}\right)=$ $\bigcup\left\{\operatorname{Pairs}(s) \mid s \in S_{1} \cup S_{2}\right\}=\alpha_{s p}\left(S_{1}\right) \cup \alpha_{s p}\left(S_{2}\right)$. Therefore $\alpha_{s p}$ is additive and so there exists $\gamma_{s p}$ such that $\left(S H, \alpha_{s p}, P S, \gamma_{s p}\right)$ forms a Galois connection. It follows that $\left(C_{L}, \alpha_{p s}, P S, \gamma_{p s}\right)$ is the composition of $\left(C_{L}, \alpha_{s h}, S H, \gamma_{s h}\right)$ and $\left(S H, \alpha_{s p}, P S, \gamma_{s p}\right)$, and so $P S$ is more abstract than $S H$.

The mirror property of $\alpha_{s h}$ is $\alpha_{s h}^{m}: C_{L} \rightarrow S H^{d}$ defined such that

$$
\alpha_{s h}^{m}(\Theta)=\bigcap_{\theta \in \Theta}\{\operatorname{occs}(\theta, x) \mid x \in \operatorname{Vars}, \operatorname{occs}(\theta, x) \neq \emptyset\} .
$$

Lemma 5. There exists $\gamma_{s h}^{m}$ such that $\left(C_{L}, \alpha_{s h}^{m}, S H^{d}, \gamma_{s h}^{m}\right)$ forms a Galois insertion.

Proof. By Theorem 1, there exists $\gamma_{s h}^{m}$ such that $\left(C_{L}, \alpha_{s h}^{m}, S H^{d}, \gamma_{s h}^{m}\right)$ forms a Galois connection. To prove $\alpha_{s h}^{m}$ is onto, we show $\forall a \in S H^{d} . \exists \theta \in S u b . \alpha_{s h}^{m}(\{\theta\})=$ $a$ by induction on $\operatorname{Card}(a)$. 
The base case is when $a=\emptyset$. Let $\theta=\{x \rightarrow t \mid x \in X\}$ where $t$ is a ground term. Then $\alpha_{s h}^{m}(\{\theta\})=\emptyset$.

Suppose $\exists s \in a$ and let $a^{\prime}=a \backslash\{s\}$. Using the induction hypothesis, $\exists \theta^{\prime} \in$ $S u b . \alpha_{s h}^{m}\left(\left\{\theta^{\prime}\right\}\right)=a^{\prime}$. Let $u \in \operatorname{Vars} \backslash X$ be a variable such that $u \notin \operatorname{var}\left(\theta^{\prime}(x)\right)$ for any $x \in X$. For every $y \in s$, suppose $\theta^{\prime}(y)=t_{y}^{\prime}$. Let $t_{y}$ be a term such that $\operatorname{var}\left(t_{y}\right)=\operatorname{var}\left(t_{y}^{\prime}\right) \cup\{u\}$. Then defining $\theta$ such that $\theta(x)=t_{x}$ for all $x \in s$ and $\theta(x)=\theta^{\prime}(x)$ otherwise, $\alpha_{s h}^{m}(\{\theta\})=a$.

The mirror property of $\alpha_{p s}$ is $\alpha_{p s}^{m}: C_{L} \rightarrow P S^{d}$ defined such that

$$
\alpha_{p s}^{m}(\Theta)=\bigcap_{\theta \in \Theta}\{\{x, y\} \in \operatorname{Pairs}(X) \mid \operatorname{var}(\theta(x)) \cap \operatorname{var}(\theta(y)) \neq \emptyset\}
$$

Lemma 6. There exists $\gamma_{p s}^{m}$ such that $\left(C_{L}, \alpha_{p s}^{m}, P S^{d}, \gamma_{p s}^{m}\right)$ forms a Galois insertion.

Proof. By Theorem [1] there exists $\gamma_{p s}^{m}$ such that $\left(C_{L}, \alpha_{p s}^{m}, P S^{d}, \gamma_{p s}^{m}\right)$ forms a Galois connection. We show that $\alpha_{p s}^{m}$ is onto.

First suppose $a=\top_{p s}=\operatorname{Pair} s(X)$. Let $u \in \operatorname{Vars} \backslash X$. Then if $\theta(x)=u$ for every $x \in X, \alpha_{p s}^{m}(\{\theta\})=\operatorname{Pairs}(X)$ as required.

Suppose $a \neq \top_{p s} . P S$ is dual-atomistic with atom $_{p s^{d}}=\{\operatorname{Pairs}(X) \backslash\{\{x, y\}\} \mid$ $\{x, y\} \in P S\}$. Therefore for every $a \neq \top_{p s}, a=\bigcap\left\{x \mid x \in\right.$ atom $\left._{p s^{d}} \wedge a \subseteq x\right\}$. But $\alpha_{p s}^{m}(\Theta)=\bigcap\left\{p_{p s}(\theta) \mid \theta \in \Theta\right\}$, and so it is sufficient to show that $\forall a \in$ atom $_{\text {ps }} . \exists \theta \in S u b . p_{\text {ps }}(\theta)=a$.

Suppose $a=$ Pairs $(X) \backslash\{\{x, y\}\}$ and let $u, v \in$ Vars $\backslash X$. Defining $\theta$ such that $\theta(x)=u, \theta(y)=v$ and $\theta(z)=f(u, v)$ for every $z \in X \backslash\{x, y\}, p_{p s}(\theta)=a$.

Theorem 3. If $\operatorname{Card}(X) \geq 3$ then $S H^{m}$ is not more precise than $P S^{m}$.

Proof. We need to show there exists $\Theta \in C_{L}$ such that $\gamma_{s h}^{m}\left(\alpha_{s h}^{m}(\Theta)\right) \nsubseteq \gamma_{p s}^{m}\left(\alpha_{p s}^{m}(\Theta)\right)$.

Suppose $X=\{x, y, z\}$ (it is easy to generalise the proof for $\operatorname{Card}(X)>$ 3). Let $\Theta=\left\{\theta_{1}, \theta_{2}\right\}$ where $\theta_{1}=\{x \rightarrow y, z \rightarrow y\}$ and $\theta_{2}=\{x \rightarrow y\}$. It follows that $\gamma_{s h}^{m}\left(\alpha_{s h}^{m}\left(\left\{\theta_{1}, \theta_{2}\right\}\right)\right)=\gamma_{s h}^{m}(\{\{x, y, x\}\} \cap\{\{x, y\}\})=\gamma_{s h}^{m}(\emptyset)=S u b$. But $\gamma_{p s}^{m}\left(\alpha_{p s}^{m}\left(\left\{\theta_{1}, \theta_{2}\right\}\right)\right)=\gamma_{p s}^{m}(\{\{x, y\}\}) \subset S u b$. Therefore $\gamma_{s h}^{m}\left(\alpha_{s h}^{m}(\Theta)\right) \nsubseteq \gamma_{p s}^{m}\left(\alpha_{p s}^{m}(\Theta)\right)$.

Thus in general the precision ordering is not preserved for mirror properties.

Theorem 4. $P S^{m}$ is not more precise than $S H^{m}$.

Proof. We need to show there exists $\Theta \in C_{L}$ such that $\gamma_{p s}^{m}\left(\alpha_{p s}^{m}(\Theta)\right) \nsubseteq \gamma_{s h}^{m}\left(\alpha_{s h}^{m}(\Theta)\right)$.

Let $\Theta=\{\epsilon\}$ where $\epsilon$ is the identity substitution. Now $\gamma_{s h}^{m}\left(\alpha_{s h}^{m}(\{\epsilon\})\right)=$ $\gamma_{s h}^{m}(\{\{x\} \mid x \in X\}) \subset S u b$ and $\gamma_{p s}^{m}\left(\alpha_{p s}^{m}(\{\epsilon\})\right)=\gamma_{p s}^{m}(\emptyset)=S u b$. Therefore $\gamma_{p s}^{m}\left(\alpha_{p s}^{m}\left(\Theta^{\prime}\right)\right) \nsubseteq \gamma_{s h}^{m}\left(\alpha_{s h}^{m}\left(\Theta^{\prime}\right)\right)$.

Hence the precision of $S H^{m}$ and $P S^{m}$ is not comparable in general. 


\section{Operations on Concrete Domains}

When the concrete lattice $C$ is join-generated by $J I(C)$, many operations on $C$ can be defined in terms of operations on $J I(C)$.

Definition 3. Suppose $C$ is join-generated by $J I(C)$. Then op is a $J I$ operation if $o p: J I(C) \times J I(C) \rightarrow J I(C)$. For each concrete operation $O p: C \times C \rightarrow C$, we say $O p$ is uniformly defined from a $J I$ operation op if for all $c_{1}, c_{2} \in C$,

$$
O p\left(c_{1}, c_{2}\right)=\bigsqcup_{C}\left\{o p\left(x_{1}, x_{2}\right) \mid x_{1}, x_{2} \in J I(C) \wedge x_{1} \sqsubseteq_{C} c_{1} \wedge x_{2} \sqsubseteq_{C} c_{2}\right\} .
$$

Example 4. In logic programming, unification and projection can both be defined as $J I$ operations unif $: S u b \times S u b \rightarrow S u b, \operatorname{proj}_{V}: S u b \rightarrow S u b$ (for $V \subseteq$ Vars) as follows:

$$
\begin{gathered}
\text { unif }\left(\theta_{1}, \theta_{2}\right)=\operatorname{mgu}\left(\operatorname{eqn}\left(\theta_{1}\right), \operatorname{eqn}\left(\theta_{2}\right)\right), \\
\operatorname{proj}_{V}(\theta)=\theta^{\prime} \text { where for each } x \in \operatorname{Vars}, \theta^{\prime}(x)= \begin{cases}\theta(x) & \text { if } x \in V \\
x & \text { otherwise }\end{cases}
\end{gathered}
$$

where $\operatorname{eqn}(\theta)=\{x=t \mid x \rightarrow t \in \theta\}$.

The concrete operations Unif: $C_{L} \times C_{L} \rightarrow C_{L}$ and $\operatorname{Proj}_{V}: C_{L} \rightarrow C_{L}$ can be uniformly defined from unif and $\operatorname{proj}$ as follows:

$$
\begin{gathered}
\operatorname{Unif}\left(\Theta_{1}, \Theta_{2}\right)=\bigcup\left\{\text { unif }\left(\theta_{1}, \theta_{2}\right) \mid \theta_{1} \in \Theta_{1} \wedge \theta_{2} \in \Theta_{2}\right\}, \\
\operatorname{Proj}_{V}(\Theta)=\bigcup\left\{\operatorname{proj}_{V}(\theta) \mid \theta \in \Theta\right\} .
\end{gathered}
$$

Given an abstract operation $O p_{D}$, we show that if $\left\langle D, O p_{D}\right\rangle$ is a complete (and therefore also correct) abstract interpretation of $\langle C, O p\rangle$, then $\left\langle D, O p_{D}^{d}\right\rangle$ is a correct abstract interpretation of $\langle C, O p\rangle$.

Lemma 7. Suppose $C, D$ are complete lattices and $C$ is join-generated by $J I(C)$. Let $O p: C \times C \rightarrow C$ be a concrete operation uniformly defined from the $J I$ operation op : $J I(C) \times J I(C) \rightarrow J I(C)$. Let $\left\langle D, O p_{D}\right\rangle$ be a complete abstract interpretation of $O p$ specified by $(C, \alpha, D, \gamma)$. Then $\left\langle D^{d}, O p_{D}\right\rangle$ is a correct abstract interpretation of $\langle C, O p\rangle$ specified by $\left(C, \alpha^{m}, D^{d}, \gamma^{m}\right)$.

Proof. We need to show that $O p\left(\gamma^{m}\left(d_{1}\right), \gamma^{m}\left(d_{2}\right)\right) \sqsubseteq_{C} \gamma^{m}\left(O p_{D}\left(d_{1}, d_{2}\right)\right)$ for all $d_{1}, d_{2} \in D$.

Note that from Definition 3 it follows that $O p$ is monotonic, i.e. if $c_{1} \sqsubseteq C c_{1}^{\prime}$ and $c_{2} \sqsubseteq_{C} c_{2}^{\prime}$ then $O p\left(c_{1}, c_{2}\right) \sqsubseteq_{C} O p\left(c_{1}^{\prime}, c_{2}^{\prime}\right)$. Since $\left\langle D, O p_{D}\right\rangle$ is complete, $O p_{D}=$ $\alpha \circ O p \circ \gamma$. Hence since $O p, \alpha, \gamma$ are all monotonic, $O p_{D}$ is also monotonic. Now

\footnotetext{
${ }^{1}$ Note that to simplify the notation we assume that a $J I$ operation has at most two input arguments. The results presented can easily be extended to operations with any number of arguments.
} 
$O p\left(\gamma^{m}\left(d_{1}\right), \gamma^{m}\left(d_{2}\right)\right)=$ $\bigsqcup_{C}\left\{o p\left(x_{1}, x_{2}\right) \mid x_{1}, x_{2} \in J I(C) \wedge x_{1} \sqsubseteq_{C} \gamma^{m}\left(d_{1}\right) \wedge x_{2} \sqsubseteq_{C} \gamma^{m}\left(d_{2}\right)\right\}$.

Therefore it is sufficient to show that $o p\left(x_{1}, x_{2}\right) \sqsubseteq_{C} \gamma^{m}\left(O p_{D}\left(d_{1}, d_{2}\right)\right)$ for all $x_{1}, x_{2} \in J I(C)$ such that $x_{1} \sqsubseteq_{C} \gamma^{m}\left(d_{1}\right)$ and $x_{2} \sqsubseteq_{C} \gamma^{m}\left(d_{2}\right)$. Now $x_{1} \sqsubseteq_{C} \gamma^{m}\left(d_{1}\right)$ implies $\alpha^{m}\left(x_{1}\right) \sqsubseteq_{D}^{d} d_{1}$ and $x_{2} \sqsubseteq_{C} \gamma^{m}\left(d_{2}\right)$ implies $\alpha^{m}\left(x_{2}\right) \sqsubseteq_{D}^{d} d_{2}$. Hence since $O p_{D}$ is monotonic,

$$
O p_{D}\left(\alpha^{m}\left(x_{1}\right), \alpha^{m}\left(x_{2}\right)\right) \sqsubseteq_{D}^{d} O p_{D}\left(d_{1}, d_{2}\right) .
$$

But $x_{1}, x_{2} \in J I(C)$, thus $O p_{D}\left(\alpha^{m}\left(x_{1}\right), \alpha^{m}\left(x_{2}\right)\right)=O p_{D}\left(\alpha\left(x_{1}\right), \alpha\left(x_{2}\right)\right)$. Since $O p_{D}$ is complete,

$$
O p_{D}\left(\alpha\left(x_{1}\right), \alpha\left(x_{2}\right)\right)=\alpha\left(O p\left(x_{1}, x_{2}\right)\right)=\alpha\left(o p\left(x_{1}, x_{2}\right)\right) .
$$

By Definition 3, $o p\left(x_{1}, x_{2}\right) \in J I(C)$ and so $\alpha\left(o p\left(x_{1}, x_{2}\right)\right)=\alpha^{m}\left(o p\left(x_{1}, x_{2}\right)\right)$. Thus $\alpha^{m}\left(o p\left(x_{1}, x_{2}\right)\right) \sqsubseteq_{D}^{d} O p_{D}\left(d_{1}, d_{2}\right)$ and so $o p\left(x_{1}, x_{2}\right) \sqsubseteq_{C} \gamma^{m}\left(O p_{D}\left(d_{1}, d_{2}\right)\right)$.

Example 5. The abstract projection function for Pos, Proj $j_{V}^{\text {pos }}:$ Pos $\rightarrow$ Pos, amounts to existentially quantifying a formula (see 6] for details). It is shown that $\left\langle\operatorname{Pos} \operatorname{Proj}_{V}^{\text {pos }}\right\rangle$ is complete in Lemma 36 [6] 2 . Therefore by Lemma 7 $\left\langle P o s^{d}, \operatorname{Proj}_{V}^{\text {pos }}\right\rangle$ is a correct abstract interpretation of $\left\langle C_{L}, \operatorname{Proj}_{V}\right\rangle$.

The abstract projection function for Sharing, $\operatorname{Proj}_{V}^{s h}: S H \rightarrow S H$, is defined such that

$$
\operatorname{Proj}_{V}^{s h}(S)=\{s \cap V \mid s \in S\}
$$

Theorem 5.2 [5] shows that $\left\langle S H, \operatorname{Proj}_{V}^{s h}\right\rangle$ is complete. Therefore by Lemma 7 $\left\langle S H^{d}, \operatorname{Proj}_{V}^{s h}\right\rangle$ is a correct abstract interpretation of $\left\langle C_{L}, \operatorname{Proj}_{V}\right\rangle$.

On the other hand, 6] shows that $\left\langle P o s, U n i f^{\text {pos }}\right\rangle$ is not complete and [5] shows that $\left\langle S H, U_{n i f}{ }^{s h}\right\rangle$ is not complete.

In fact, it can be shown that both $\left\langle P o s^{d}, U n i f^{\text {pos }}\right\rangle$ and $\left\langle S H^{d}, U n i f^{s h}\right\rangle$ are not correct abstract interpretations of $\left\langle C_{L}, U n i f\right\rangle$.

Lemma 8. $\left\langle P o s^{d}, U n i f^{\text {pos }}\right\rangle$ is not a correct abstract interpretation of $\left\langle C_{L}, U n i f\right\rangle$.

Proof. It is sufficient to find $\phi \in \operatorname{Pos}^{d}$ such that

$$
\operatorname{Unif}{ }^{\text {pos }}(\phi, \phi) \not \models \alpha_{\text {pos }}^{m}\left(U n i f\left(\gamma_{\text {pos }}^{m}(\phi), \gamma_{\text {pos }}^{m}(\phi)\right)\right) \text {. }
$$

Let $\phi$ be the formula $x \leftrightarrow y$ and $\theta_{1}=\{x \rightarrow f(1, y)\}$ and $\theta_{2}=\{x \rightarrow f(y, 1)\}$. Note that $\theta_{1}, \theta_{2} \in \gamma_{\text {pos }}^{m}(\phi)$. Now unif $\left(\theta_{1}, \theta_{2}\right)=\{x \rightarrow f(1,1), y \rightarrow 1\}$ and so it follows that

$$
\alpha_{\text {pos }}^{m}\left(\operatorname{Unif}\left(\gamma_{\text {pos }}^{m}(\phi), \gamma_{\text {pos }}^{m}(\phi)\right)\right) \models x \wedge y .
$$

But $U n i f^{\text {pos }}(\phi, \phi)=\phi$ and so $U n i f^{\text {pos }}(\phi, \phi) \not \models \alpha_{\text {pos }}^{m}\left(U n i f\left(\gamma_{\text {pos }}^{m}(\phi), \gamma_{\text {pos }}^{m}(\phi)\right)\right)$, as required.

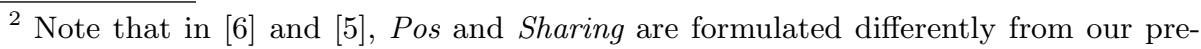
sentation. In [6] and [5], however, it is evident that the proofs can be adapted.
} 
Lemma 9. $\left\langle S H^{d}, U n i f^{s h}\right\rangle$ is not a correct abstract interpretation of $\left\langle C_{L}, U n i f\right\rangle$.

Proof. It is sufficient to find $S \in S H^{d}$ and a binding $x \rightarrow t$ such that

$$
U n i f^{s h}(S, x \rightarrow t) \nsubseteq \alpha_{s h}^{m}\left(U n i f\left(\gamma_{s h}^{m}(S),\{\{x \rightarrow t\}\}\right)\right) \text {. }
$$

Let $S=\{\{x, y\}\}, t=f(1, y)$ and $\theta=\{x \rightarrow f(y, 1)\}$. Note that $\theta \in \gamma_{s h}^{m}(S)$. Now unif $(\theta,\{x \rightarrow t\})=\{x \rightarrow f(1,1), y \rightarrow 1\}$ and so it follows that

$$
\alpha_{s h}^{m}\left(U n i f\left(\gamma_{s h}^{m}(S),\{\{x \rightarrow t\}\}\right)=\emptyset\right.
$$

But $U n i f^{s h}(S, x \rightarrow t)=\{\{x, y\},\{x\},\{y\}\}$ and so the result follows.

Hence new abstract unification operations need to be devised for both Pos $^{d}$ and $S H^{d}$.

\section{Conclusion}

We have shown how, given an abstract domain $D$ specifying a lattice property $\alpha_{p}$, an abstract domain $D^{d}$ specifying the mirror property $\alpha_{p}^{m}$ can be constructed. We have also shown that if $\left\langle D, O p_{D}\right\rangle$ is a complete abstract interpretation of $\left\langle C, O p_{C}\right\rangle$, then $\left\langle D^{d}, O p_{D}\right\rangle$ is a correct abstract interpretation of $\left\langle C, O p_{C}\right\rangle$.

There are instances when non-complete abstract operations computing a property can be used to improve the precision of operations computing the mirror property. For example, formulae of the form $x \rightarrow y$ in Pos are interpreted as meaning " $x$ ground implies $y$ ground". The contrapositive of this is " $y$ nonground implies $x$ non-ground". Thus this information could be used to improve the precision of a $\operatorname{Pos}^{d}$ analysis. In fact, since non-groundness information is approximated by freeness information, it would seem reasonable to implement Pos $^{d}$ as a reduced product construction with Pos and a domain expressing freeness information. It would be interesting to see if generalisations of this method could be meaningfully applied to other domains. Another direction for future work is to see how our approach relates to lower/upper approximations used in concurrency 17.

\section{Acknowledgments}

We thank the anonymous referees for their useful comments. This work was supported by EPSRC Grant GR/M05645.

\section{References}

1. R. Bagnara, P. M. Hill, and E. Zaffanella. Set-sharing is redundant for pair-sharing. In P. Van Hentenryck, editor, Static Analysis: Proceedings of the 4th International Symposium, volume 1302, pages 53-67, Paris, France, 1997. 
2. R. Balbes and P .Dwinger. Distributive Lattices. University of Missouri Press, Columbia, Missouri, 1974.

3. G. Birkhoff. Lattice Theory. AMS Colloquium Publication, Providence, RI, 3rd edition, 1967.

4. M. Codish, A. Mulkers, M. Bruynooghe, M. García de la Banda, and M. Hermenegildo. Improving abstract interpretations by combining domains. ACM Transactions on Programming Languages and Systems, 17(1):28-44, 1995.

5. A. Cortesi and G. Filè. Sharing is Optimal. Journal of Logic Programming, 38(3):371-386, 1999.

6. A. Cortesi, G. Filè, and W. Winsborough. Optimal Groundness Analysis Using Propositional Logic. Journal of Logic Programming, 27(2):137-167, 1996.

7. P. Cousot and R. Cousot. Abstract Interpretation: A Unified Lattice Model for Static Analysis of Programs by Construction or Approximation of Fixpoints. In Proc. Fourth ACM Symp. Principles of Programming Languages, pages 238-252, 1977.

8. P. Cousot and R. Cousot. Systematic Design of Program Analysis Frameworks. In Proc. Sixth ACM Symp. Principles of Programming Languages, pages 269-282, 1979.

9. P. Cousot and R. Cousot. Abstract Interpretation and Applications to Logic Programs. Journal of Logic Programming, 13(2 \& 3):103-179, 1992.

10. R. Giacobazzi and F. Ranzato. Refining and Compressing Abstract Domains. In Proceedings of the 24th International Colloquium on Automata, Languages and Programming ICALP 97, volume 1256 of Lecture Notes in Computer Science, pages 771-781. Springer-Verlag, 1997.

11. R. Giacobazzi and .F Ranzato. Optimal Domains for Disjunctive Abstract Interpretation. Science of Computer Programming, 32:177-210, 1998.

12. R. Giacobazzi, F. Ranzato, and F. Scozzari. Making Abstract Interpretations Complete. Journal of the ACM. (to appear).

13. R. Giacobazzi, F. Ranzato, and F. Scozzari. Building Complete Abstract Interpretations in a Linear Logic-based Setting. In G. Levi, editor, Static Analysis, Proceedings of the Fifth International Static Analysis Symposium SAS 98, volume 1503 of Lecture Notes in Computer Science, pages 215-229. Springer-Verlag, 1998.

14. R. Giacobazzi and F. Scozzari. A Logical Model for Relational Abstract Domains. ACM Transactions on Programming Languages and Systems, 20(5):10671109, 1998.

15. P. Hill and F. Spoto. Freeness Analysis through Linear Refinement. In Static Analysis: Proceedings of the 6th International Symposium, volume 1694, pages 85100, 1999.

16. N.D. Jones and H. Søndergaard. A Semantics-based Framework for the Abstract Interpretation of Prolog. In S. Abramsky and C. Hankin, editors, Abstract Interpretation of Declarative Languages, pages 123-142. Ellis Horwood Ltd, 1987.

17. F. Levi. A Symbolic Semantics for Abstract Model Checking. In Static Analysis: Proceedings of the 5th International Symposium, volume 1503, pages 134-151, 1998.

18. F. Scozzari. Logical Optimality of Groundness Analysis. In P. Van Hentenryck, editor, Proceedings of International Static Analysis Symposium, SAS'97, volume 1302 of Lecture Notes in Computer Science, pages 83-97. Springer-Verlag, 1997. 